\title{
A Kerr Electro-Optical Technique for Observation and Analysis of High-Intensity Electric Fields *
}

\author{
Esther C. Cassidy and Harold N. Cones \\ Institute for Basic Standards, National Bureau of Standards, Washington, D.C. 20234
}

(May 19, 1969)

\begin{abstract}
A Kerr electro-optical technique, which permits observation and analysis of high intensity electric fields in nitrobenzene-filled Kerr cells, is described. Two-dimensional visual images, similar to those achieved in photoelastic-mechanical stress analysis, of the field distribution are afforded by the fringe pattern produced by the Kerr effect when high direct voltages are applied to the cell. Analysis of the field profile, by measurement of the fringe positions, permits calibration of the system for measurement of high voltage pulses. The Kerr constant of the liquid and space-resolved determinations of relative field strength, actual field strength (in volts per centimeter), and potential are also derived.
\end{abstract}

Key words: Dielectric liquids; electro-optical measurements; electrostatic field measurements; high voltage measurements; Kerr cell; laser applications; potential measurements; pulse measurements.

\section{Introduction}

The present study was conducted in order to determine the distribution and relative intensity of the electric field imposed by application of high direct voltage across the electrodes of nitrobenzene-filled Kerr cells, our ultimate goal being the development of methods for accurate calibration of Kerr-type high-voltage pulse-measuring systems [1]. ${ }^{1}$ Except for edge effects, the electric field between parallel-plate electrodes should be uniform in intensity, directly proportional to the applied voltage, and inversely proportional to the interelectrode distance. If these conditions were actually achieved, the Kerr pulse-measuring technique would be straightforward, and calibrations of the system could be achieved by both direct- and pulsevoltage divider techniques [2]. However, it is evident from the literature [3] that the field between high voltage electrodes immersed in a dielectric liquid is often distorted by the presence of free charges in the liquid, under both dc and pulse conditions. Since these socalled "space charges" are believed to be either ionic impurities present in the liquid before imposition of the field or ions formed in consequence of the field, it would seem that field distortion effects might be reduced by using ultra-pure liquids in the Kerr cell. Although liquids of adequate purity are not available commercially, several sophisticated methods for achieving a high-degree of purity have been reported

*This werk was supported in part by the U.S. Atomic Energy Commission through the Sandia Corporation, Albuquerque, New Mexico.

' Figures in brackets indicate the literature references at the end of this paper.
[4-10]. However, in spite of use of several of the more effective purification techniques, recent studies [2, 11-13] indicate continued distortion in the field distribution, particularly when high-intensity d-c fields $(>15 \mathrm{kV} / \mathrm{cm})$ are applied.

This paper reports development of a method for high direct-voltage calibration of Kerr systems suitable for measuring pulses peaking as high as $100 \mathrm{kV}$ in spite of nonuniformity in the interelectrode field due to space charge effects. In addition to achievement of this immediate goal, the experimental techniques employed are believed to be unique in that they permit direct observations, similar to those achieved in photoelastic mechanical-stress analysis, of the behavior of distorted high-intensity electrostatic fields between electrodes immersed in a dielectric liquid. In the present work, the Kerr cells were filled with nitrobenzene. However, it is evident that other dielectric liquids with reasonably high Kerr constants may be studied by applying higher voltages and adjusting the cell geometry.

Unlike earlier methods $[2,14-16]$, the present technique provides the following advantages: (1) It permits direct observation of the two-dimensional field distribution. The regions subject to greatest electrical stress, where breakdowns are more likely to occur, may therefore be detected by direct observation. This advantage has not, to the authors' knowledge, been fully utilized in earlier Kerr effect measurements' of this type, perhaps because voltages of sufficient magnitude and/or electrodes of suitable length were not employed. (2) It enables determination of field strength (in volts per centimeter) and potential. 
Such measurements were often achieved in earlier work $[13,17]$ by inserting potential probes in the liquid. The present Kerr electro-optical technique avoids the disturbing influence of the probes. (3) These values may be determined, with any given high direct voltage applied, from a single photograph of the light transmitted by the interelectrode area. Point by point measurements of the transmitted light intensity are not required. Measurement resolution increases with the magnitude of the applied field. (5) Use of a laser light source allows simplicity in the optical system.

\section{Apparatus and Procedure}

The experimental setup is shown schematically in figure 1. A laser beam is passed through and along the

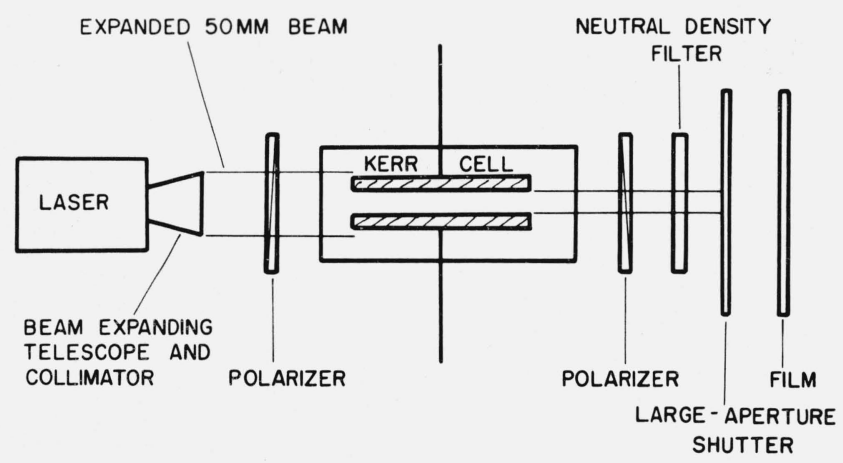

FIGURE 1. The Kerr electro-optical system with laser source and recording photographic film.

length of a Kerr cell, equipped with parallel-plate nickel electrodes and filled with high purity nitrobenzene. The cell is installed between two polarizers. The first polarizer is oriented so that the laser light entering the cell is linearly polarized at an angle of $45^{\circ}$ with respect to the parallel surfaces of the electrodes. The second polarizer (the analyzer) is "crossed" with respect to the first, so that initially no light is passed by the system. When voltage is applied across the Kerr cell, the imposed electric field induces birefringence in the nitrobenzene. Thus, when light passes between the electrodes of the cell, its state of polarization is altered by the action of the electric field, producing the so-called Kerr effect [18].

Measurement of the intensity of the light transmitted by the analyzer has long been used for study of electric fields in dielectric liquids which become birefringent in the presence of an electric field. Moller [19], who determined the field distribution by photometric scanning of the light transmitted by the interelectrode area, was among the first to report its use for this purpose. In later works $[7,11,15,20-23]$ retardation plates and compensators were inserted in the light path, in order to make photographic recording of the field distribution more feasible. In some cases, collimating lenses and filters, which restricted the radiation passed to very narrow wavelength regions, were also included. Since nonmonochromatic light sources were used, the filter served to reduce errors resulting from the wavelength dependence of the Kerr constant [15].

In the present work, the latter difficulty is avoided by using a laser source (continuous-wave helium-neon, wavelength $632.8 \mathrm{~nm}$ ). Lenses are used to expand and collimate the $2.5 \mathrm{~mm}$ beam diameter to $50 \mathrm{~mm}$. A pinhole aperture is inserted at the focal point of the expanding lens to eliminate spatial noise and thereby produce a smooth intensity profile across the collimated beam. These components are housed in a beam expanding telescope attachment affixed to the laser. Except for the laser and this attachment, the Kerr system is simple and conventional. As voltage is applied to the cell, the distribution of the electric field over the entire interelectrode area is observed directly by viewing the alternate bright and dark bands or fringes produced by the Kerr effect on a translucent paper or ground glass screen inserted in the path of the light transmitted by the analyzer. Permanent, full-scale images of the electrodes and of the fieldinduced fringes may be recorded photographically for precise measurement purposes. A negative lens may be inserted between the analyzer and the photographic plate or film to obtain an enlarged image. This procedure is especially useful for direct viewing of the field between closely spaced $(<1 \mathrm{~cm})$ electrodes. The results shown in figures $2-7$ were obtained using neutral density filters which transmitted 1 to 25 percent of the light transmitted by the analyzer. Exposure times ranged from 1 to $10 \mathrm{~s}$.

\section{Analysis of the Electric Field}

The intensity $I$ of the light transmitted by the analyzer upon application of voltage to the Kerr cell is related to the strength $E$ of the imposed field as follows $[1,2,25]$ :

$$
\frac{I}{I_{m}}=\sin ^{2}\left[\frac{\pi}{2}\left(\frac{E}{E_{m}}\right)^{2}\right]
$$

where $I_{m}$ is the maximum light intensity transmitted by the system, and $E_{m}$ is the electric field strength which produces the first transmission maximum. Thus, if the field is uniform, the light intensity transmitted by the analyzer will change uniformly over the entire interelectrode area as the voltage across the cell is changed. When the imposed field $E=E_{m}$, the first transmission maximum will be observed. As the voltage is increased further, transmission will decrease until $E=\sqrt{2} E_{m}$. At this point (the first transmission minimum), the entire interelectrode region will be dark on the viewing screen. With further increases in voltage, the transmitted light intensity will continue to oscillate between maximum and minimum transmission points, the interelectrode area observed on the viewing screen being brightest when $E=E_{m}, \sqrt{3} E_{m}$, $\sqrt{5} E_{m}$, etc., and darkest when $E=0, \sqrt{2} E_{m}, 2 E_{m}$, etc.

However, if the electric field is nonuniform, as is often the case when high direct voltages are applied 
across electrodes immersed in a dielectric liquid $[3,12,19]$, the intensity of the light transmitted by the analyzer will vary with the average local field strength along the length of each light path through the liquid according to eq (1). When fields of sufficient strength are applied, the Kerr effect will produce extreme variations (alternate bright and dark bands), similar to those observed by Mueller [26] under low ac-field conditions, in the light intensity transmitted over the interelectrode area.

The photographs in figure 2 were taken using the Kerr system described above with the indicated high direct voltages applied across a nitrobenzene-Kerr cell having parallel plate electrodes (dimensions: $1.5 \mathrm{~cm} \times 10 \mathrm{~cm} \times 0.2 \mathrm{~cm})$ and interelectrode spacing $d=1 \mathrm{~cm}$. As the voltage was increased, alternate bright and dark fringes appeared, thus permitting direct observation of the profile of the electric field across the width of the interelectrode area. ${ }^{2}$ Regions where

${ }^{2}$ Errors in the profile due to the fringing fields at the end edges of the electrodes are believed to be small if the interelectrode distance is small compared with the length of the electrodes.
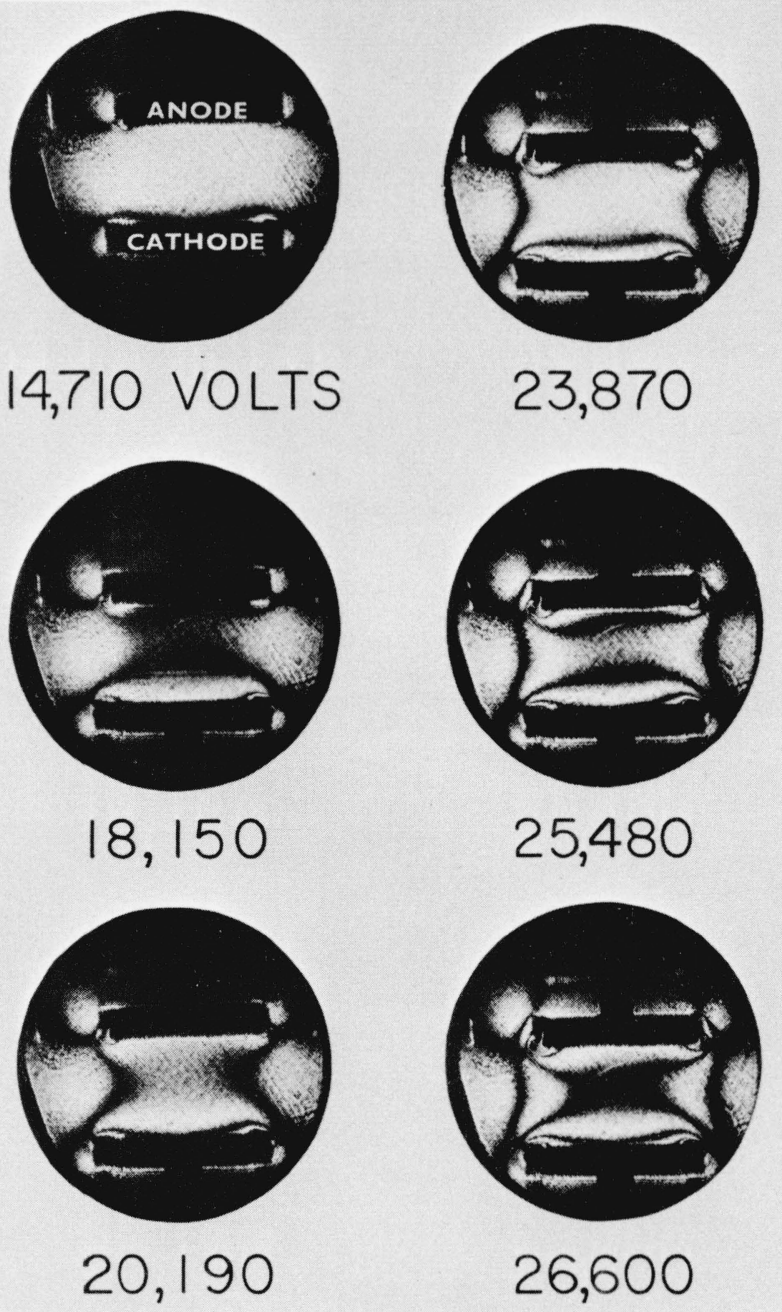

FIGURE 2. Photographs showing dark fringes (minimum transmission bands) produced by Kerr effect when high direct voltages are applied

to a nitrobenzene Kerr cell $(\mathrm{d}=1 \mathrm{~cm})$.

the field distortion is greatest are evidenced by concentrations of a greater number of fringes. The photographs of figure 2 show immediately that the field in this cell is most intense and very nonuniform at the edges of the electrodes. Since the existence of fringing field edge effects is well known, some distortion was expected in these regions. However, the distortion indicated by the fringes appearing in the central interelectrode area, which is attributed to space charge effects, is difficult to predict. Further, the fringes indicate that the interelectrode field in this cell is stronger near the electrodes and weaker in the region midway between them. It may also be noted that the interelectrode field is more intense near the cathode when voltages lower than $32,000 \mathrm{~V}$ are applied. At higher voltages, the greater number of fringes at the anode indicates that the interelectrode field is more intense near the anode.

In the present work, successive numerical values were assigned to each successive bright $(n=1,3,5$, etc.) and dark $(n=2,4,6$, etc.) band as it appeared in consequence of the increasing voltage, according to
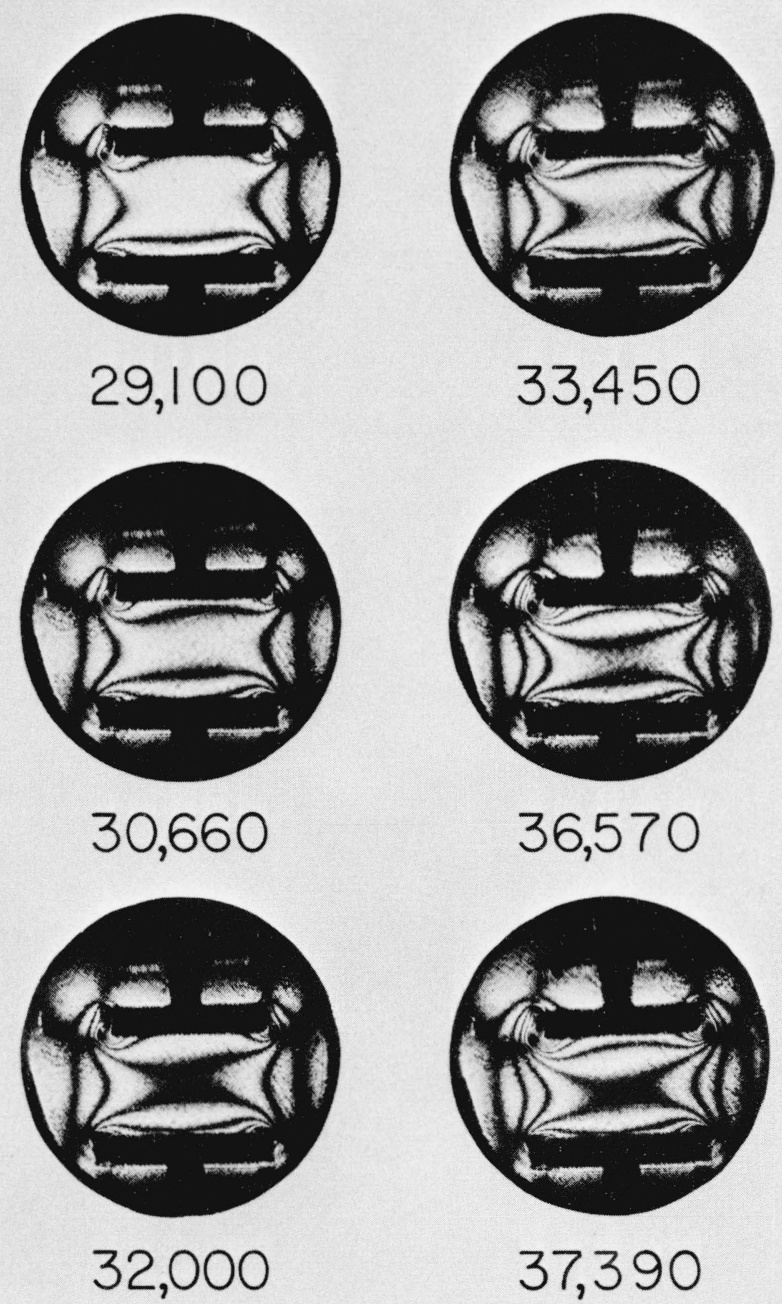
the convention described in our earlier work [2]. The dark fringes recorded in the last photograph of figure 2 , with 37.390 volts applied to the cell, are numbered in this manner in figure 3 . As the voltage was increased, the first dark fringes to appear at each electrode are assigned the value $n=2$, the second to appear $n=4$, and so on. As may be seen from figure 2 , the fringes appeared initially at each electrode, moved away from their respective electrodes toward the center of the cell where they merged, then divided and moved off to the outer perimeter of the Kerr cell wall.
The relative field strength $\left(E / E_{m}\right)$ along the equifield line delineated by the brightest and darkest points, where $\left(I / I_{m}\right)=1$ and $\left(I / I_{m}\right)=0$, respectively (see eq (1)), of each bright and dark band is related to the assigned $n$ value as follows [2]:

$$
\left(\frac{E}{E_{m}}\right)=\sqrt{n}
$$

If a voltage high enough to produce several (five or more) dark fringes in the interelectrode area is applied,

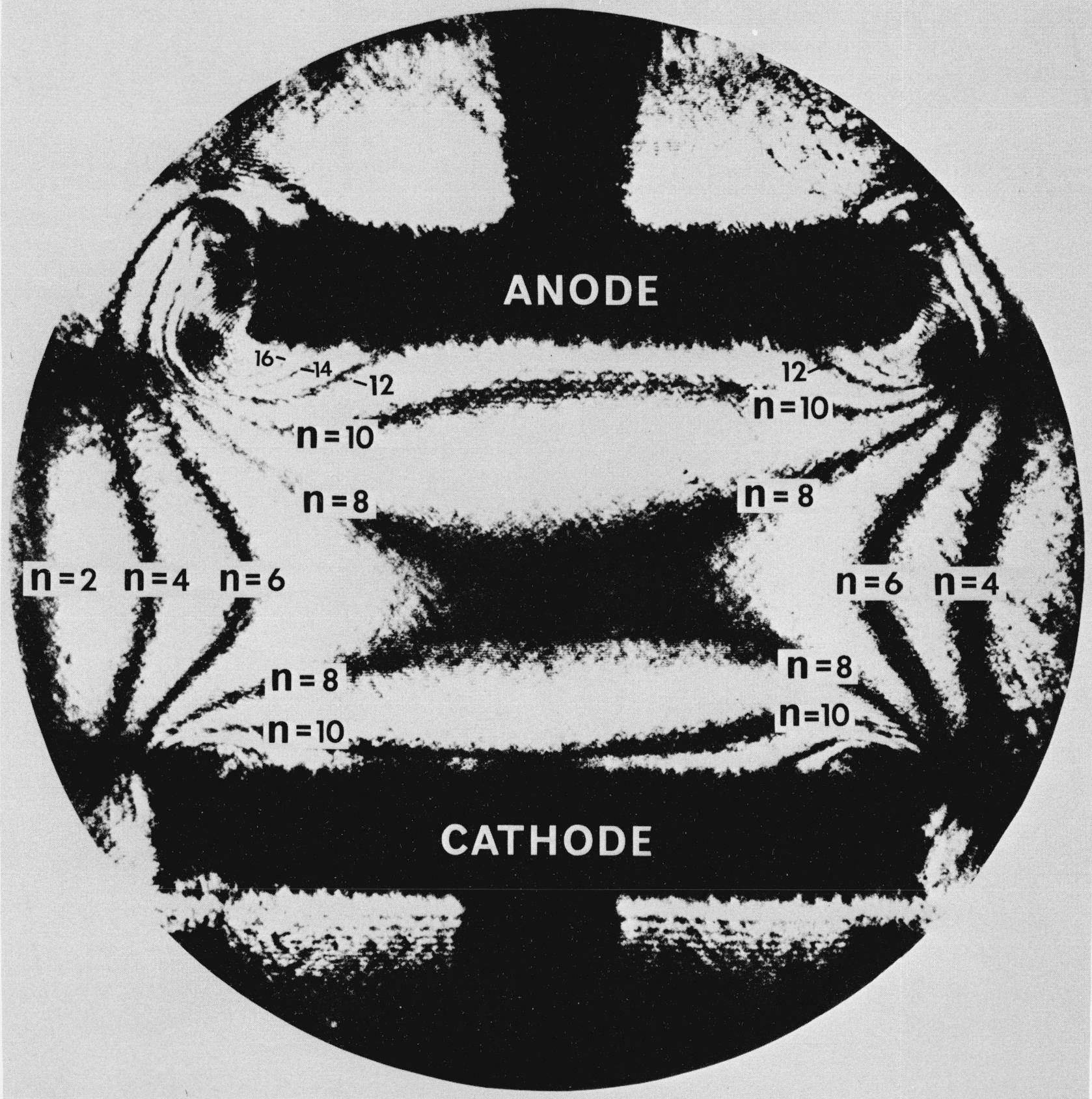

FIGURE 3. Enlarged photograph with numerical values assigned to the equifield lines delineated by the dark fringes. Applied voltage $=37,390 \mathrm{~V}$. 
the relative field strength $\left(E / E_{m}\right)$ may be plotted as a function of distance (from one of the electrodes) simply by measuring the positions of the dark fringes. Quantitive measurements of the transmitted light intensity were found to be unnecessary. The curves in figure 4 , which show the relative field strength

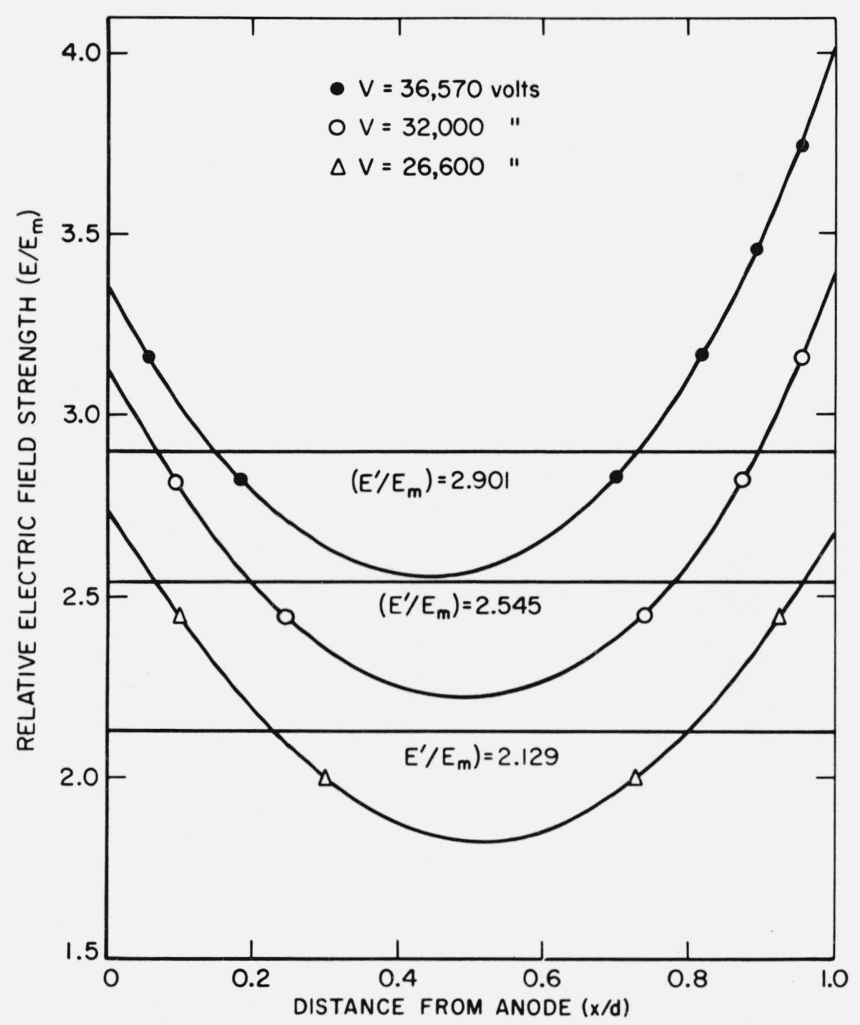

FIGURE 4. The nonuniform electric field distributions and average relative field strengths $\left(\mathrm{E}^{\prime} / \mathrm{E}_{\mathrm{m}}\right)$ as determined from measurements of fringe positions with various voltages applied.

$\left(E / E_{m}\right)$ as a function of distance (from the anode), with three different voltages applied to the cell, were plotted in this manner using three of the photographs in figure 2. The fringe positions, indicated by the points on the curves, were determined from measurements made by several methods: (1) by tracing and measuring the position of enlarged projections of the dark fringes and electrodes on fine-scale graph paper, (2) by measuring their positions on enlargements of the original photograph with a millimeter scale, and (3) from microdensitometer measurements of the position of the darkest point in each fringe on transparent film.

Similar photographs showing the electric field distribution imposed by different voltages in a nitrobenzene Kerr cell having $12 \mathrm{~cm}$ long parallel electrodes and interelectrode spacing $d=2.76 \mathrm{~cm}$ are given in figure 5. In this cell, the field distortion is more pronounced. If the photographs are viewed in sequence with the increasing applied voltage, it is also evident that the behavior of the field differs from that observed in the $1 \mathrm{~cm}$ cell. In this case, numerous dark fringes appeared in sequence at the cathode and moved across the interelectrode distance as the voltage was increased. As the first dark fringe $(n=2)$ moved near the anode, it divided into three sections, two of which moved off to the outer perimeter of the cell. The other section moved into the anode. Although voltages higher than $70 \mathrm{kV}$ were not applied in the present work, we anticipate that this behavior would continue as the voltage is increased. Since the electrode spacing is believed to be the principal difference between this cell and the $1 \mathrm{~cm}$ cell, the evident contrast in their field distributions cannot as yet be fully explained. Further work investigating this and the extent of fringing field errors is now in progress.

The relative strength $\left(E / E_{m}\right)$ of the electric field in the $2.76 \mathrm{~cm}$ cell, as determined from measurements of the positions of the dark fringes in the photograph (in fig. 5) taken with $65,920 \mathrm{~V}$ applied, is plotted as a function of distance from the anode in figure 6 . The enlarged photograph used for these measurements, with successive numerical values assigned to the fringes as described above, is shown in figure 7 .

\section{Calibration of System for Measurement of High Voltage Pulses}

The average relative field strength $\left(E^{\prime} \mid E_{m}\right)$ imposed by an applied voltage $V$ may be determined from the relative field distribution measurements by numerical integration of the following equation

$$
\left(\frac{E^{\prime}}{E_{m}}\right)=\int_{0}^{1}\left(\frac{E}{E_{m}}\right) d\left(\frac{x}{d}\right)
$$

along a central line perpendicular to the inner surfaces of the electrodes. ${ }^{4}\left(E / E_{m}\right)$ is the relative local field intensity read, as a function of normalized position $(x / d)$, from the measured field distribution curves (e.g., those of figs. 4 and 6).

Going further, it is evident that $\left(E^{\prime} / E_{m}\right)$ is the relative strength of the uniform field which the applied voltage $V$ would impose in a given Kerr cell. One may therefore write

$$
\frac{V}{\left(E_{m} d\right)}=\left(\frac{E^{\prime}}{E_{m}}\right)
$$

where $\left(E_{m} d\right)$ is the voltage required to produce the first transmission maximum in a given cell under uniform field conditions. $V$ may be measured to within 0.01 percent by use of a high-direct-voltage divider [24], and $\left(E^{\prime} / E_{m}\right)$ may be derived as described above. Equation (4) therefore permits determination of the cell constant $\left(E_{m} d\right)$ required in calibration of the Kerr system for measurement of high voltage pulses, provided, of course, that the fields imposed by the pulses to be measured are uniform. ${ }^{5}$ At a temperature of $24^{\circ} \mathrm{C}$, the cell constant of the $1 \mathrm{~cm}$ cell used in the

\footnotetext{
${ }^{4}$ In this central interelectrode region, the field direction is perpendicular to the electrode surfaces.

5 In view of the experimental results of Croitoru [23], assumption of uniformity in the pulse field would seem to be reasonable for pulses with duration less than $50 \mu \mathrm{s}$.
} 
present work was found to be $12,530 \mathrm{~V}$. This value was averaged from results obtained by measuring the fringe positions with different voltages between 26,000 and $37,000 \mathrm{~V}$ applied to the cell. As may be seen from table 1 , the calculated cell constants were identical to within 1 percent. Because of their reproducibility

TABLE 1. Comparison of pulse divider calibration [2] with direct voltage calibrations of $1 \mathrm{~cm} \mathrm{Kerr} \mathrm{cell}$

\begin{tabular}{c|c|c|c}
\hline $\begin{array}{c}\text { Pulse Divider } \\
\text { Calibration }\end{array}$ & \multicolumn{3}{|c}{ Direct Voltage Calibrations and Data } \\
\hline$\left(E_{m} d\right)$ & $V$ & $\left(E^{\prime} / E_{m}\right)$ & $\left(E_{m} d\right)$ \\
\hline \multirow{2}{*}{ Volts } & Volts & & Volts \\
& 26,600 & 2.129 & 12,500 \\
12,560 & 30,660 & 2.445 & 12,540 \\
& 32,000 & 2.545 & 12,570 \\
& 33,450 & 2.677 & 12,500 \\
& 36,570 & 2.919 & 12,530 \\
\hline
\end{tabular}

and close agreement with calibrations achieved by one of the pulse divider methods described earlier [2], the present calibrations and the measurements used in achieving the calibration are believed to be accurate to within 1 percent.

\section{Measurement of Other Parameters}

In addition to the above, the field intensity $E_{m}$ (in volts per centimeter) which produces the first transmission maximum in the liquid under study may be determined from the following relationship,

$$
E_{m}=\frac{V}{\left(E^{\prime} / E_{m}\right) d}
$$

by measurement of the interelectrode distance $d$. $V$ and $\left(E^{\prime} / E_{m}\right)$ are measured as described above. Then, since
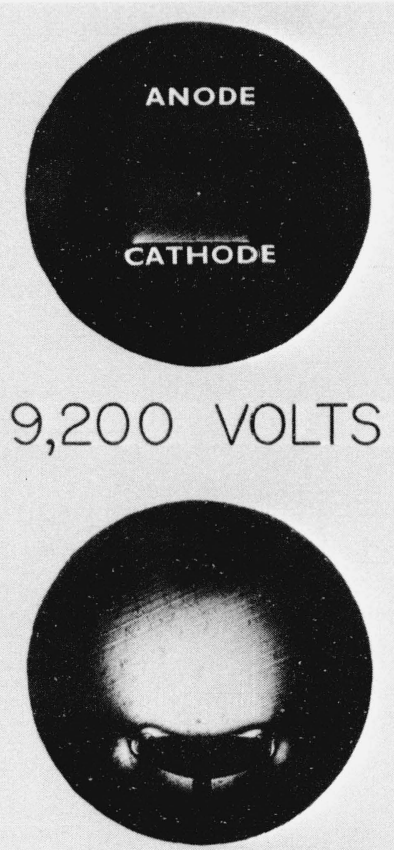

15,840

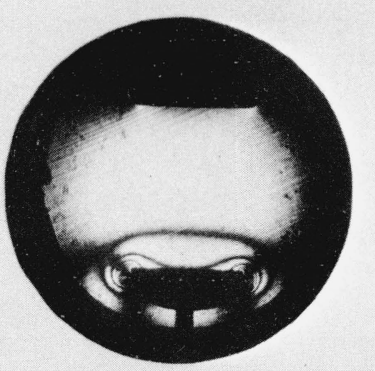

26,290

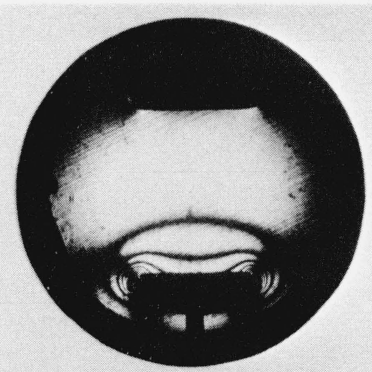

37,340

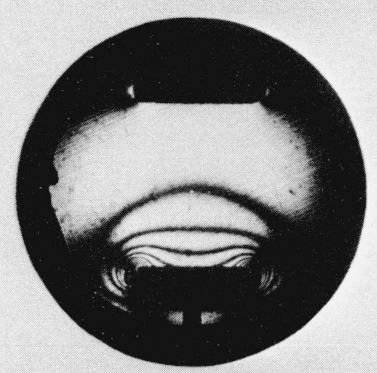

$$
47,160
$$

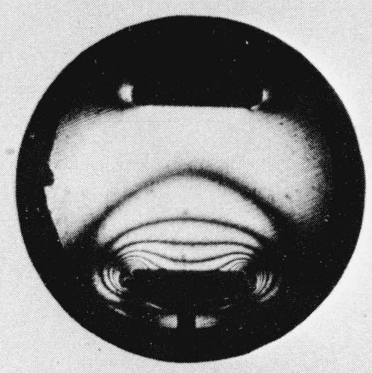

53,920
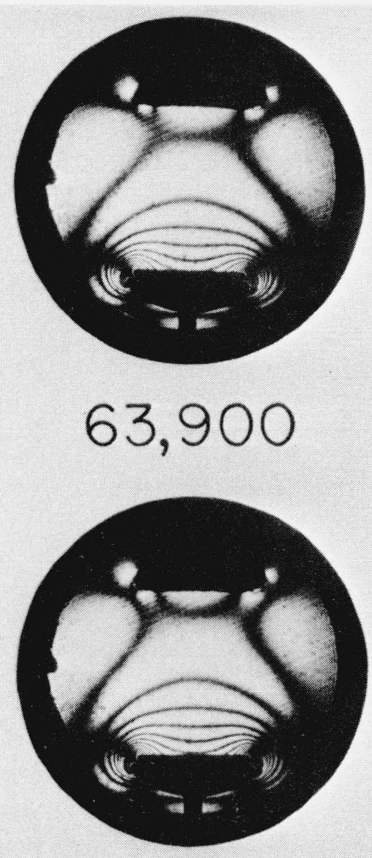

$$
65,920
$$

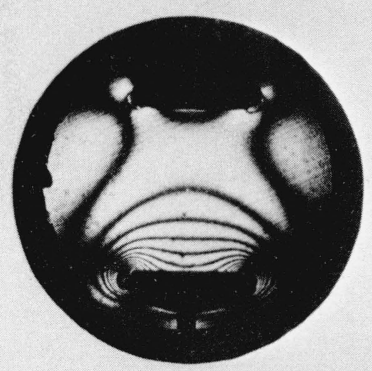

68,960

FIGURE 5. Photographs of Kerr effect fringes produced in $2.76 \mathrm{~cm}$ nitrobenzene cell at various voltages. 


$$
B=\frac{1}{2 l E_{m}^{2}},
$$

the Kerr constant $B$ of the liquid may be obtained by measurement of the length $l$ of the light path through

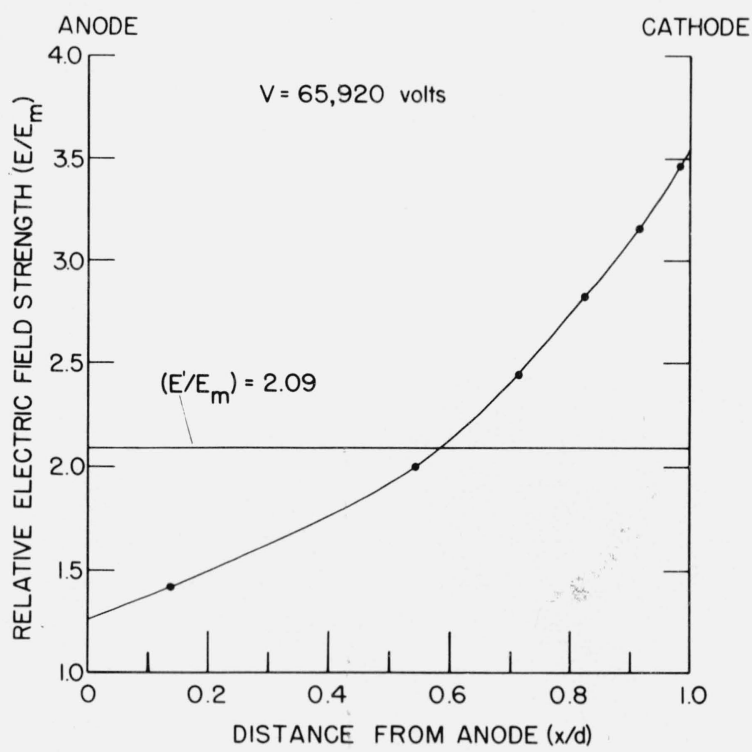

FIGURE 6. The electric field distribution between the inner central surfaces of the electrodes in the $2.76 \mathrm{~cm}$ cell.

the electric field. Results obtained with the $1 \mathrm{~cm}$ cell indicate the Kerr constant of nitrobenzene at a temperature of $24{ }^{\circ} \mathrm{C}$ and wavelength of $682.8 \mathrm{~nm}$ to be about $3 \times 10^{-5}$ esu. Since the distance between the electrodes of this cell is small compared with their length $(10 \mathrm{~cm})$, the errors due to edge effects at the ends of the electrodes are believed to be small. An end effect correction was therefore not included in this calculation. If corrections for the wavelength and temperature dependence of $B$ are applied, the determined value compares favorably with those reported in the literature [15].

Further, since the field intensity $E_{m}$ required to produce maximum transmission is constant throughout the liquid, the local field intensity $E_{x}$ (in volts per centimeter) at any point $x$ may be determined from

$$
E_{x}=\left(\frac{E}{E_{m}}\right) E_{m}
$$

by measuring the fringe positions along the perpendicular interelectrode line which passes through the point. The relative field strength $\left(E / E_{m}\right)$ at the point $x$ is then read from the relative field distribution curve as described above. Similarly, the potential $V_{x}$ at any point $x$ along a line perpendicular to the inner surfaces of the electrodes and parallel to the field direction may be derived from the local field intensities as follows

$$
V_{x}=\int_{0}^{x} E_{x} d x
$$

If such detailed information on the field and potential distribution is not required, it is important to note that the field intensity profile is mapped directly in two dimensions by the dark fringes. Each dark fringe $n$ delineates an equifield line of relative intensity $\left(E / E_{m}\right)_{n}$, with $\left(E / E_{m}\right)_{n}$ being known directly from the numerical value $n$ by use of eq (2). Then, with $E_{m}$ for the liquid being known from eq (5), an actual intensity $E_{n}$ in volts per centimeter may be assigned to each fringe by substituting these values in eq (7).

\section{Summary and Conclusions}

A technique, which utilizes laser light for Kerr electro-optical analysis of the distorted electric field imposed when high direct voltages are applied to electrodes immersed in some dielectric liquids, has been described. The laser beam (50 mm diam) is directed between the electrodes so that the entire interelectrode area is illuminated. The nonuniform distribution of the electric field is immediately evident from direct observations of the dark fringes produced by the Kerr effect in consequence of the gradients in the high-intensity field. Since each fringe delineates an equifield line of known relative strength $\left[\left(E / E_{m}\right)=\sqrt{2}, 2, \sqrt{6}\right.$, etc. $]$, the field strength along each fringe is determined by counting the fringes as they appear upon applications of voltages of increasing magnitude.

The cell constant, required in calibration of the system for measurement of those short pulse voltages which produce uniform field distributions, is calculated from the relative field distribution by measurement of the applied direct voltage. Comparison of calibrations derived from these measurements with those derived from conventional pulse-divider measurements indicate that the field measurements are accurate within 1 percent. The field strength $E_{m}$ which produces maximum transmission in the liquid, the Kerr constant of the liquid, and the potential at any point along lines perpendicular to the electrodes and parallel to the field direction are among other parameters which may be derived from photographic records of the transmitted fringe pattern.

The experimental results demonstrate the accuracy $(\sim 1 \%)$ of the quantitative measurements achieved by use of the Kerr technique described here. A further advantage appears to be the two-dimensional visual image of the electric field distribution afforded by the transmitted interelectrode fringe pattern. As in photoelastic mechanical-stress analysis, regions of highest electrical stress are evidenced by concentrations of a greater number of fringes, thus enabling immediate detection of these regions where electrical breakdown is more likely to occur. In addition, the technique provides a means for direct observation of the influence of alterations in the shape and geometry of the cell and its electrodes. We anticipate using it for this purpose in the near future. Finally, though such observations must be made in liquids having reasonable Kerr constants and dielectric strengths, the authors 
hope that the technique described will lead to a better understanding of the behavior of high-intensity electric fields in other liquid insulants.

The authors are grateful to F. Ralph Kotter, F. L. Hermach, and I. R. Bartky of the National Bureau of Standards and to S. R. Booker of Sandia Corporation for helpful discussions and suggestions, to William Bagley for assistance in purification of the nitrobenzene and to Mrs. Mary Hull for assistance in the mathematical calculations.

\section{References}

[1] Wunsch, D. C., and Erteza, Z., Kerr cell measuring system for high voltage pulses, Rev. Sci. Instr. 35, pp. 816-820 (1964).

[2] Cassidy, E. C., Cones, H. N., Wunsch, D. C., and Booker, S. R., Calibration of a Kerr cell system for high voltage pulse measurements, IEEE Trans. on Instrumentation and Measurement IM-1 7, pp. 313-320 (1968).

[3] Croitoru, Z., Progress in Dielectrics 6 (Space charge in dielectrics), pp. 105-111 and pp. 121-124 (J. B. Birks and J. Hart Ed., Academic Press, Inc., N.Y., 1965).

[4] White, H. J., The technique of Kerr cells, Rev. Sci. Instr. 6, pp. 22-26 (1935).

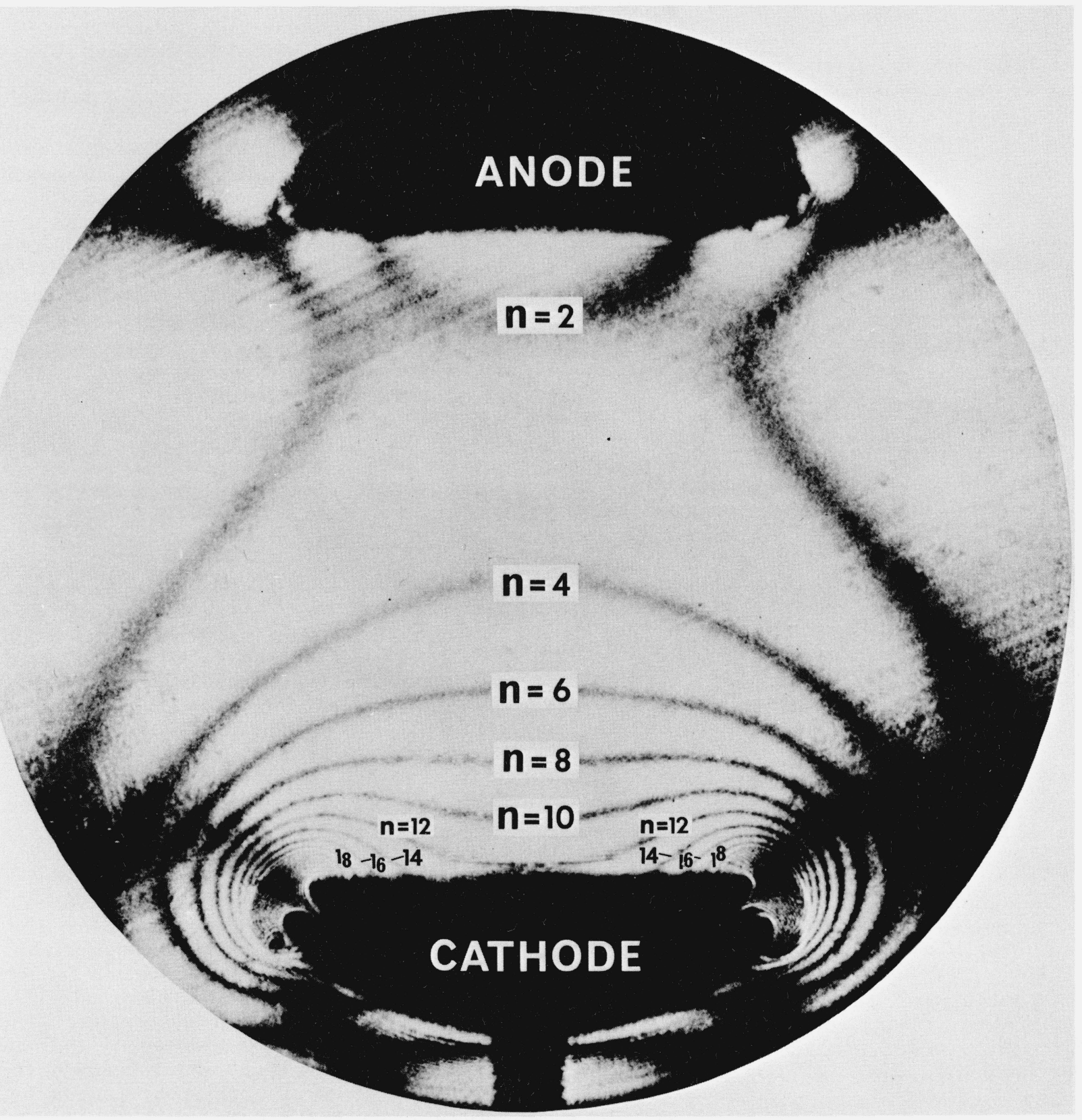

FigURE 7. Kerr effect fringes, with numerical values assigned, in $2.76 \mathrm{~cm}$ cell. Applied voltage $=65,920 \mathrm{~V}$. 
[5] Cheshko, F. F., Bydylo, L. K., Naumenko, V. P., and Shevchenko, O. I., Purification of nitrobenzene, trans. from Zhurnal Prikladnoi Khimii 34, pp. 908-916 (1961).

[6] Felici, N. J., The de-ionization of strongly polar liquids, Brit. J. Appl. Phys. 15, pp. 801-805 (1964).

[7] Jeanmaire, G., Etude experimentale des phenomenes de polarisation dan les dielectriques, Revue Generale de L'Electricite 74, pp. 515-516 (1965).

[8] Briere, G., Felici, N., and Filippini, J. C., Desionisation du nitrobenzene par electrodialyse, C. R. Acad. Sc. Paris 261, pp. 5097-5099 (1965).

[9] Briere, G., and Rose, B., Electrodialyse des solvants polaires. Purification ionique des liquides aprotiques, J. Chim. Physique 64, pp. 1720-1725 (1967).

[10] Blanchet, M., Proc. 8th Internat. Cong. on High-Speed Photography (Dispositif pour la determination de la constante de Kerr des liquids. Proprietes de noveaux liquids electrooptiques. Installation de remplissage de cellules de Kerr.) in press.

[11] Briere, G., and Gosse, J. P., Electrodialyse des solvants polaires. Epuration ionique du nitrobenzene controlee par observation simultanee de la repartition du champ electrique et de l'evolution du courant en fonction du temps. J. Chim. Physique 65, pp. 1341-1348 (1968).

[12] Briere, G., and Gaspard, F., Electric conduction in nitrobenzene, Chem. Phys. Letters 1, pp. 706-708 (1968).

[13] Taris, F., and Guizonnier, R., Conductibilite des liquid isolants sous tensions continues de 20 a $100 \mathrm{kV}$, Revue Generale de L'Electricite 75, pp. 1295-1299 (1966).

[14] Croitoru, Z., reference 3, pp. 131-137 (1965).

[15] Moller, R., Die Kerrkonstante des Nitrobenzols, Physikalische Zeitschift 32. pp. 697-718 (1931).
[16] Beams, J. W., Electric and magnetic double refraction, Rev. Mod. Phys. 4, pp. 137-139 (1932).

[17] Whitehead, J. B., and Marvin, R. H., The conductivity of insulating oils, Trans. AIEE 49, pp. 647-656 (1930).

[18] Kerr, J., A new relation between electricity and light: Dielectrified media birefringent, Phil. Mag. 50, pp. 337-348 and 446-458 (1875).

[19] Moller, R., Die kerr-konstante des nitrobenzols, Physikalische Zeitschrift 30, pp. 20-24 (1929).

[20] Hehlgans, F., Beitrage zur physik der nitrobenzolkerrzelle, Physikalische Zeitschrift 32, pp. 718-727, 803-808, 951-957 and 971-974 (1931).

[21] Goodwin, D. W., Space charge phenomena in liquid dielectrics, Proc. Phys. Soc. B69, pp. 61-69 (1956).

[22] Hill, C. E., and House, H., Measurement of electric field distribution in $n$-Hexane using an optical probe, Electronics Letters 3, pp. 78-79 (1967).

[23] Croitoru, Z., Colombeáu, M., and Coelho, R., 1961 Annual Report Conf. on Electrical Insulation (Space charges in liquid dielectric), pp. 21-24, Publication 973, Nat'l. Acad. of Sciences-Nat'l. Research Council, Washington, D.C. (1962).

[24] Park, J. H., Special shielded resistor for high-voltage d-e measurements, J. Res. NBS 66C (Eng. and Instr.), No. 1, pp. 19-24 (1962).

[25] Zarem, A. M., Marshall, F. R., Poole, F. L., An electro-optical shutter for photographic purposes, Elect. Eng. 68, pp. 282288 (1949).

[26] Mueller, H., Electro-optical field mapping, J. Opt. Soc. Am. 31, pp. 286-291 (1941).

(Paper 73C1 \& 2-284) 